PACS: 03.67.-2; 73.23.Ad; 75.47.Jn; 85.35.Ds

\title{
Spin ballistic transport and quantum interference in mesoscopic loop structures
}

\author{
I. Tralle and W. Paśko \\ Institute of Physics, University of Rzeszów, 16A, Al. Rejtana, 35-310 Rzeszów, Poland \\ E-mail: tralle@univ.rzeszow.pl
}

\begin{abstract}
In the paper, a simple theory of quantum inteference in a loop structure caused by spin coherent transport and the Larmor precession of the electron spin is presented. A "spin ballistic" regime is supposed to occur, when the phase relaxation length for the spin part of the wavefunction $\left(L_{\varphi}^{(s)}\right)$ is much greater than the phase relaxation length for the "orbital part" $\left(L_{\varphi}^{(e)}\right)$. In the presence of an additional magnetic field, the spin part of the electron wavefunction acquires a phase shift due to additional spin precession around that field. If the structure length $L$ is chosen to be $L_{\varphi}^{(s)}>L>L_{\varphi}^{(e)}$, it is possible to "wash out" the quantum interference related to the phase coherence of the "orbital part" of the wavefunction, retaining at the same time that related to the phase coherence of the spin part and, hence, to reveal corresponding conductance oscillations. Different mechanisms of spin relaxation, such as Elliot - Yafet, the scattering by the edges and surface the structure and the precession ones, as well as their influence on the spin coherent transport are considered. The quantum interference in time-dependent magnetic field, quantum beats in mesoscopic loop structure, are also discussed. The similarities between this effect and Josephson, scalar Aharonov - Bohm and Aharonov - Casher effects, as well as their differences are treated and possible application of the effect to the construction of the device, complementary to superconducting quantum interference device is analyzed.
\end{abstract}

Keywords: spin ballistic transport, mesoscopic structures, quantum interference, spintronics, Josephson effect, scalar Aharonov - Bohm and Aharonov - Casher effects.

Manuscript received 10.02.05; accepted for publication 18.05.05.

\section{Introduction}

The majority of all modern electronic devices are based solely upon the transport of electron charge, quantum transport including. And yet charge is not the only distinguishing feature of the electron, another one is its spin. Despite the contemporary focus on semiconductor quantum devices, this second principal quantum mechanical aspect of the electron, - its spin has largely been ignored. However, in recent years a new paradigm of electronics based on the spin degree of freedom of the electron has began to emerge, so even the name for this new branch of the science and technology was coined, spintronics. Plainly speaking, spintronics is an attempt to substitute the electron charge by its spin in order to use it for the variety of practical applications [1]. The single biggest boost to the field of spintronics has been the discovery of the Giant Magneto-Resistance (GMR) and GMR-sandwich structures. GMR was discovered in 1988 in a superlattice with alternating $\mathrm{Fe}$ and $\mathrm{Cr}$ layers [2] and this effect has become the basis for a majority of spintronic devices. Up to now most of attention was paid to manipulation of electron spins in micro- and nanostructures by means, for instance, spin injection [3], while another possible non-classical devices based on quantum inteference of spins attracted less attention.

One of the main ideas which underpins various possible applications of "spin transport", including information storage and computation, is that the spins of electrons in semiconductors may have very long quantum coherence times [4], or in other words, electrons can travel a long way without flipping their spins. But this also gives the possibility to observe quantum effects which involve the interference of electron waves. In the classical picture of transport phenomena, the total probability for a particle to transfer from one point to another is the sum of the probabilities for such transfer over all possible trajectories. In the quantum description, this result corresponds to neglecting the interference of scattered electron waves propagating along different paths. The destruction of quantum coherence is controlled by the phase relaxation time or phase relaxation length. Since for the electron spin this length may be very long, it is naturally to expect that the spin interference can reveal itself in the conductance oscillations similar to those caused by the 
Aharonov - Bohm (AB) effect. Most of the researchers who dealt with the $\mathrm{AB}$ effect in the solid state [5] considered mainly the Hamiltonian $\hat{H}=(\mathbf{p}-(e / c) \mathbf{A})^{2} / 2 m^{*}+U(y, z)$, where $U(y, z)$ is the energy corresponding to the transverse motion, and almost nobody takes into account the spin-part $\mu_{\mathrm{B}} \hat{\sigma} \mathbf{B}$ of the Hamiltonian ( $\mu_{B}$ is the Bohr magneton, $\hat{\sigma}$ is the electron spin operator, $\mathbf{B}$ is the magnetic field). However, if the quantum interference is concerned, the quantity of main importance is the coherence length. If one considers the total Hamiltonian that includes the Pauli term, one can write down the electron wavefunction in a factorized form as the tensor product: $\Psi(r, s)=\varphi(r) \otimes \chi(s)$ and consider the coherence of each part separately. As a result, it is possible to introduce two phase relaxation lengths, the first one for the ,orbital part" of the electron wavefunction, $L_{\varphi}^{(e)}$, and the second one, $L_{\varphi}^{(s)}$ for the spin part of the wavefunction. It turns out $[6-8]$ that $L_{\varphi}^{(s)}>>L_{\varphi}^{(e)}$ which is in total agreement with the experiment [4]. The physics which is behind that is the following. An electron during its transfer along some path in the solid (semiconductor, for definiteness) interacts all the time with the environment. As a rule, rigid scatters such as impurities and other defects of crystalline structure do not contribute to the phase relaxation; only dynamical scatters like phonons do. On the other hand, the electron scattering by phonons is mainly inelastic, while impurity scattering is mainly elastic, so we can say that only inelastic scattering contributes to the phase relaxation. But what does it mean inelastic scattering in case of spin? It means spin flips caused by spin-orbit interaction accompanied by phonon interaction, since there must be an agent which adds or subtracts the Zeeman energy to the electron spin. This kind of interaction is very weak and that is why the spin flips are rare events and the phase relaxation length for the spin part of the electron wavefunction is very long. But now, if the structure length $L$ is chosen to be $L_{\varphi}^{(s)}>L>L_{\varphi}^{(e)}$, it is possible to "wash out" the quantum interference related to phase coherence of the "orbital part" of the wavefunction retaining at the same time the phase coherence of the spin part one and hence, to reveal the corresponding conductance oscillations of the microstructure. Such model was considered in the papers $[6,7]$, where the simple theory of the quantum interference in a loop structure due to the Larmor precession of electron spin in semiconductor microstructure was presented for the first time. The aim of this paper is to summarize our previous results, discuss other aspects of the problem, for instance, the precession mechanisms of spin relaxation which are the characteristic ones for the crystals and heterostructures with lack of inversion symmetry, as
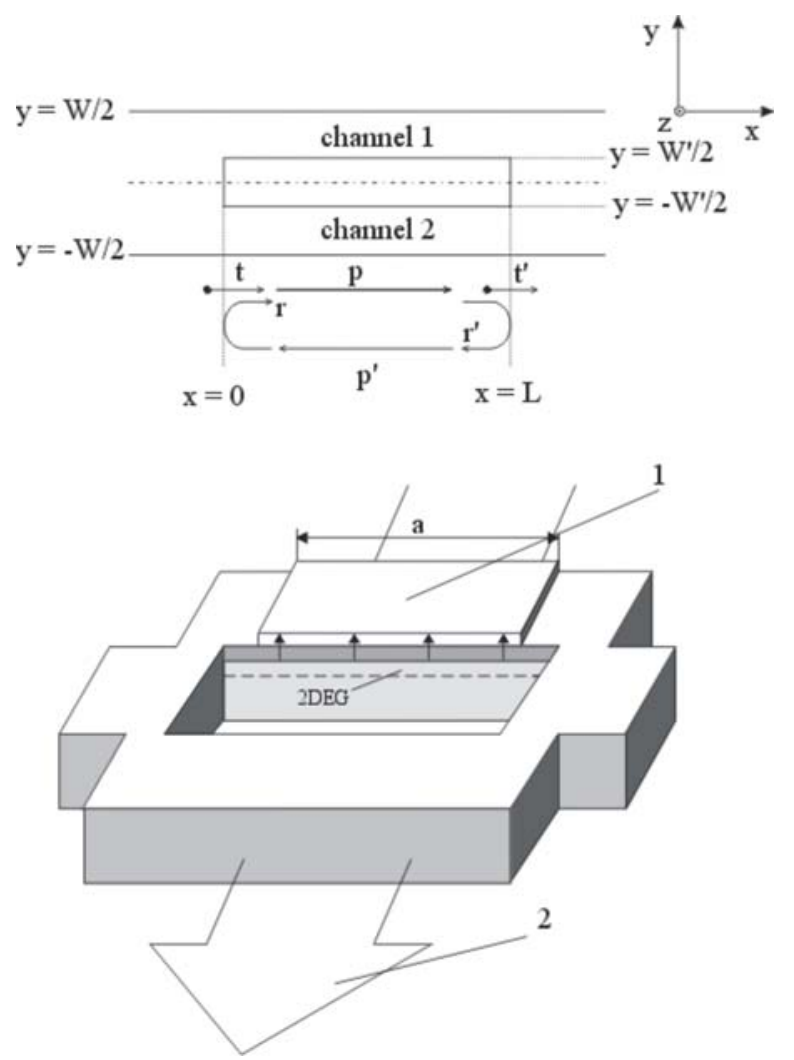

Fig. 1. Sketch of a two-channel semiconductor mesoscopic structure with an additional magnetic field accross one of the channels. On the upper panel $t, t^{\prime}, r, r^{\prime}$ indicate the transmission and reflection matrices at the two junctions $x \leq 0, x \geq L ; P, P^{\prime}$ stand for the propagation matrices in the middle region $(0 \leq x \leq L)$; the external magnetic field $B_{0}$.

well as to discuss the quantum interference of spins occurring in the time-dependent magnetic field.

\section{Elliot - Yafet mechanism of spin relaxation}

We start with a loop microstructure with two end regions $x<0$ and $x>L$ and a middle region $0 \leq x \leq L$ consisting of two channels (Fig. 1), similar to those considered in [6, 7].

Consider an electron entering the domain occupied by the magnetic field, say, from the left-end region. The electron wavefunction is a coherent superposition of the spin-up $\chi(\mid \uparrow>)$ and spin-down $\chi(\mid \downarrow>)$ eigenstates, which are split in the magnetic field by the Zeeman energy $\Delta \varepsilon=g \mu_{\mathrm{B}} B,(g$ is the Lande factor $)$. Coherent evolution under the spin Hamiltonian results in oscillations between these two eigenstates; classically this oscillation corresponds to precession of the spin vector at the Larmor frequency $\Delta \varepsilon / \hbar$. In other words, we consider the non-relativistic electron motion in the magnetic field as the motion of a classical top which precesses about the magnetic field. Since the magnetic fields are different in the two arms of the structure, the 
phase shifts acquired by the spin wavefunctions are also different.

Suppose the Hamiltonian of an electron is $H=H_{0} \otimes I_{0}+H_{1} \otimes I_{1}$, where:

$$
H_{0}=1 / 2 m^{*}(p-(e / c) A)^{2}+U(r), H_{1}=-\mu \hat{\sigma} \cdot \mathbf{B} .
$$

Here $I_{0}, I_{1}$ are the unit operators acting in the state spaces of $H_{0}$ and $H_{1}$, respectively, $m^{*}$ is the electron effective mass, $\mathbf{A}$ is the vector potential corresponding to the magnetic field $\mathbf{B}, \mu_{\mathrm{B}}$ and $\hat{\sigma}$ are Bohr magneton and the spin operator, respectively. We also assume that $U(\mathbf{r})$ describes conduction bands bending due to space charge and discontinuities of any band. Since $H_{0}$ does not depend on spin, the wavefunction is the tensor product: $\Psi(\mathbf{r}, t, s)=\varphi(\mathbf{r}, t) \otimes \chi(s, t)$. Ever since for convenience we shall refer to $\varphi(\mathbf{r}, t)$ as the "orbital part" of the total wavefunction, keeping in mind that it corresponds to $H_{0}$ describing the charge-field interaction, and we shall refer to $\chi(s, t)$ as the spin-part of the wavefunction related to $H_{1}$, the spin part of the Hamiltonian (1).

Introducing the factorized form of the wavefunction as the tensor product of orbital and spin parts, one not only can, but rather have to introduce simultaneously two phase relaxation lengths, the first one for the "orbital part" of the electron wavefunction, $L_{\varphi}^{(e)}$, and the second one, $L_{\varphi}^{(s)}$ for the spin part. As we already mentioned in Sec.1, it turns out $[6,8]$ that $L_{\varphi}^{(s)}>L_{\varphi}^{(e)}$, which is in total agreement with the experiment [4]. Physics hidden behind that is as follows. An electron during its transfer along some path in the solid (semiconductor, for definiteness) interacts all the time with the environment. As a rule, rigid scatterers such as impurities and other defects of crystalline structure do not contribute to the phase relaxation, since according to general principles, quantum coherence of the wavefunction cannot be destroyed due to elastic interactions with a static external potential. But inelastic interactions may and in general do destroy the phase coherence of the wavefunction. Let us look at the interaction of an electron with the crystal lattice from the electron reference frame. In the electron reference frame, it is the ions that constitute the crystal lattice are moving. These moving ions produce some magnetic field that acts upon electron spin and this is spin-orbit interaction mentioned in the Introduction. On the other hand, in order a spin-flip may to occur, there must be an agent which adds energy to the electron or carries it away. This agent is nothing else but phonon. So, the spin-flip is caused by spin-orbit interaction accompanied by phonon scattering. This kind of interaction (this mechanism of spin relaxation is termed in the literature as Elliot - Yafet (EY) mechanism) is weak, and that is why the spin flips are rare events.

In order to estimate the spin phase relaxation time $\tau_{\varphi, \mathrm{ph}}^{(s)}$ due to interaction with phonons, consider a simple model which actually was considered in [6] and which, for the sake of consistency and the readers' convenience, we outline here very briefly.

Let us take a two-state quantum system (which we shall refer to as subsystem $A$ ) with excitation energy $\varepsilon$ interacting with a phonon bath, and identify two states with the "spin-up" and "spin-down" states of a spin in an external magnetic field. For simplicity, we suppose the interaction of the subsystem $A$ with the phonons to be resonant; this means that only those modes of the phonon bath whose energy is equal to $\varepsilon$ interact with the two-level subsystem. Other modes are taken into account indirectly by choosing all mean values of phonon bath parameters to be equal to their statistical average at given temperature $T$. As a result, for the model of phonon bath we can take a great number $(N>>1)$ of identical non-interacting subsystems $B_{n}$ with excitation energy $\varepsilon$.

Then the Hamiltonian of the entire system (subsystem $A+$ phonon bath) is

$H=\varepsilon\left(a^{\dagger} a+\sum_{n=1}^{N} b_{n}^{\dagger} b_{n}\right)$

where $a^{\dagger}, a$ are the Fermi creation and anihilation operators related to the excitations of subsystem $A$, while $b^{\dagger}, b$ are the Bose creation and anihilation operators related to the excitations of the $n$-th subsystem of phonon bath.

Thus, for the subsystems of the equidistant spectra, we have

$$
\begin{aligned}
& \left\langle b_{n}^{\dagger} b_{n}\right\rangle=\operatorname{Sp}\left(\rho b_{n}^{\dagger} b_{n}\right)=(\exp (\beta \varepsilon)-1)^{-1}, \\
& \beta=1 / k_{\mathrm{B}} T,
\end{aligned}
$$

where $\rho_{n}$ is the statistical operator for the $B_{n}$ subsystems, $k_{\mathrm{B}}$ is the Boltzmann constant, $T$ is the temperature, $\mathrm{Sp}(\ldots)$ is the trace operator.

The interaction of two-level systems with phonons can be described by the term $H_{\text {int }}(t)$ :

$$
H_{\mathrm{int}}(t)=\sum_{n=1}^{N}(\theta(t-\tau(n-1))-\theta(t-\tau n)) H_{n},
$$

where

$$
\begin{aligned}
& \theta=\left\{\begin{array}{lll}
1 & \text { if } \quad t>0 \\
0 & \text { if } \quad t \leq 0
\end{array}\right. \\
& H_{n}=\varepsilon_{\text {int }}\left(a^{\dagger} b_{n}+b_{n}^{\dagger} a\right)
\end{aligned}
$$

and $\varepsilon_{\text {int }}$ is the interaction energy.

The physical meaning of (3) is that the subsystem $A$ interacts each time during interval $\tau$ with those subsystem $B_{n}$ which did not interact with $A$ during previous time interval, or, in other words, $\tau$ is the electron-phonon collision time.

The kinetic equation for the density matrix corresponding to Hamiltonian (1) with the interaction 
between two subsystem of the form (3), is solved in our paper [6], where also the next formula for $\tau_{\varphi, p h}^{(s)}$ is derived:

$\tau_{\varphi, \mathrm{ph}}^{(s)} \sim\left(\hbar^{2} / \tau \varepsilon_{\mathrm{int}}^{2}\right) \ln 2 \cdot \tanh (\beta \varepsilon / 2)$,

where $\varepsilon_{\text {int }}$ is an interaction energy characterizing an interaction between two-level quantum subsystem and the phonon bath.

In the work [6], only the lower boundary for $\tau_{\varphi, p h}^{(s)}$ was established, which nevertheless turned out to be much greater than $\tau_{\varphi}^{(e)}$, the phase relaxation time of the orbital part of the wavefunction. It is possible to get more rigorous theory which could make more precise calculations possible (for details see, for instance, [8] and the next Section).

Anyway, if the electron scattering by phonons is concerned, we have shown that indeed one can choose the structure length to be $L_{\varphi}^{(s)}>>L_{\varphi}^{(e)}$.

\section{Precession mechanisms of spin phase relaxation}

In the works [7, 8], we considered the edge and surface scattering and their influence on the spin phase relaxation. We have shown that this kind of scattering practically does not contribute to the "phase memory" destruction and can be neglected. The reason why the spin relaxation due to edge and surface scattering is even great much times weaker than due to EY mechanism is quite lucid: being the rigid scatters, such as impurities and other defects of crystalline structure, the edges and the surface of the structure do not contribute to the phase relaxation, since according to general principles, quantum coherence of the wavefunction cannot be destroyed due to elastic interactions with a static external potential.

So far our assertion, $L_{\varphi}^{(s)}>L_{\varphi}^{(e)}$ was proved to be valid for two possible mechanisms of spin phase relaxation: EY process and the spin relaxation due to surface scattering. However, there are other possible causes for the destruction of "phase memory" of the spin part of electron wavefunction. These are, so-called precession mechanisms of spin-relaxation, for instance, D'yakonov - Perel (DP) mechanism [9]. This mechanism is characteristic one for the crystals of zincblende structure whose point group has no inversion symmetry: in a material with bulk inversion asymmetry (BIA) the electron energy bands are spin split for a given direction of the wavevector $\mathbf{k}$. There is also another very similar mechanism which is characteristic for the heterostructures and 2DEG layers, where the spin splitting may occur as a result of the structure inversion asymmetry (SIA) and which was first pointed out by Yn. Bychkov and E. Rashba [10].

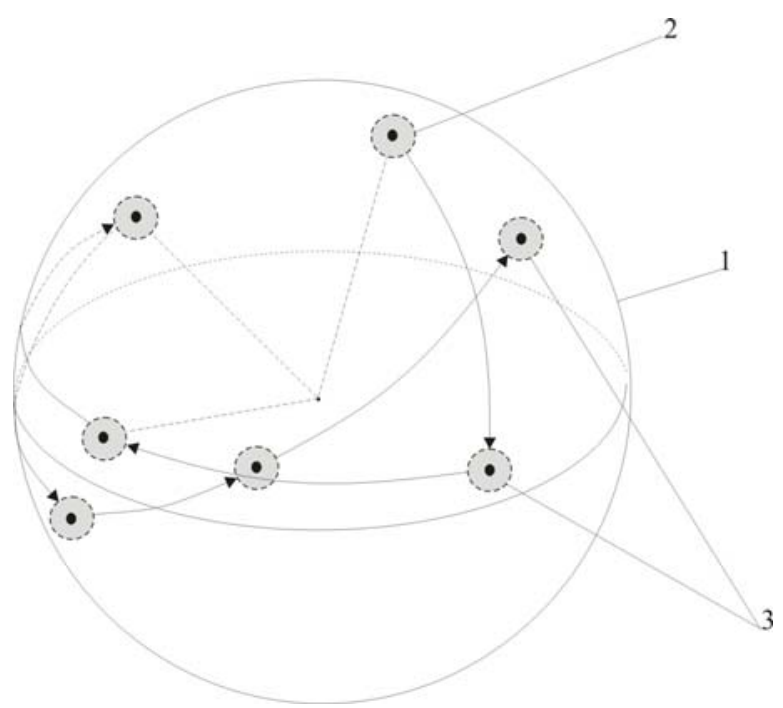

Fig. 2. Schematic representation of the precession mechnism of spin relaxation: $1-S^{2}$-sphere; 2 - a point on the $S^{2}$-sphere corresponds the the initial position of the spin precession axis; 3 - shaded cirlces correspond to the sequential positions of the spin precession axis whose direction changes randomly due to collisions.

Let us start with the DP mechanism. As is known, the diamond-type lattice consists of two similar facecentered cubic sublattices mutually penetrating each other. The zinc-blende type crystal lattices $\left(A^{\mathrm{III}} B^{\mathrm{V}}\right.$-type, for example) differ from the diamond-type one in that respect, that two sublattices are not identical: the first one consists of the $A$-type atoms, while another sublattice is of the $B$-type atoms. As a result, the point group of the zinc-blende structure does not involve the inversion and, as a consequence, the periodic part of the Bloch function satisfies no longer the condition $U_{-\mathbf{k}}(\mathbf{r})=U_{\mathbf{k}}(-\mathbf{r})$. Hence, a twofold degeneracy is lifted, and the electron energy bands are spin-split in these materials for a given direction of the wavevector $\mathbf{k}$, even if the external magnetic field is not present. As a result, another mechanism of spin relaxation proposed for the first time in [10] becomes possible. We call this mechanism as the "precession" mechanism of spin relaxation.

The spin splitting in $k$-point is equal $[9,11]$ :

$\Delta \varepsilon_{\mathbf{k}}=\eta\left(\mathbf{n}_{0}\right) k^{3}$,

$\mathbf{n}_{0}=\mathbf{k} / k$,

where $\eta\left(\mathbf{n}_{0}\right)=\eta_{0}|\kappa(\mathbf{n})|, \quad \kappa_{x}=n_{0 x}\left(n_{0 y}^{2}-n_{0 z}^{2}\right)$ and the other components of $\kappa$ can be obtained by means of cyclic permutation of indices.

The spin relaxation time could be estimated as follows. Let the initial electron state characterized by $\mathbf{k}$ be polarized along some axis, say, $a$, which does not coincide with $\kappa(\mathbf{n})$ and let its spin state be $\sigma=+1 / 2$. Regarding the spin splitting $\Delta \varepsilon_{\mathbf{k}}$, this state is not longer the eigenstate and, as time passes by, it changes. It 
means, as it was already mentioned above, that another component with $\sigma=-1 / 2$ mixes up to the state with $\sigma=+1 / 2$. The coherent mixture of these two states corresponds, in the classical picture, to the spin precession about the $\kappa(\mathbf{n})$-axis with the frequency $\Omega_{\mathbf{k}}=\Delta \varepsilon_{\mathbf{k}} / \hbar$.

The spin relaxation arises due to the momentum relaxation which is always present to some extent and accompanies the spin precession. Since $\Omega_{\mathbf{k}} \tau_{\mathbf{p}}<<1$, during the elapse of time between two successive scattering events the electron spin revolves only by a small angle $\sim \Omega_{\mathbf{k}} \tau_{\mathbf{p}}$. At each scattering event the wavevector $\mathbf{k}$ changes randomly and as a result, $\kappa(\mathbf{n})$ changes too. It means that the direction of spin precession axis changes randomly also, as the electron moves through the crystal (see Fig. 2). After many scattering events, the initial "spot"corresponding to the initial direction of the precession axis spreads over the unit sphere $S^{2}$; so, one can treat this process as some kind of the diffusion over $S^{2}$ with the angular diffusion coefficient $D_{s}$ of about $\sim\left(\Omega_{\mathbf{k}} \tau_{\mathbf{p}}\right)^{2} / \tau_{\mathbf{p}}$. The time $T$ which is needed for the initial "spot" to run over $S^{2}$ uniformly, can be estimated as $\left(D_{S} T\right)^{1 / 2} \sim 4 \pi$, and hence,

$$
T \sim(4 \pi)^{2} / \Omega_{\mathbf{k}}^{2} \tau_{\mathbf{p}}
$$

Intuitively it is clear that $T>>\tau_{\mathbf{p}}$, since one need to have many scattering events, in order the initial "spot" to run over entire $S^{2}$.

More precise calculations (see [12]) give the following formula for $\Omega_{\mathbf{k}}$ :

$$
\begin{aligned}
& \Omega_{\mathbf{k}}^{2}(\varepsilon)=\frac{16}{315}\left(\frac{\Delta}{\hbar}\right)\left(\frac{A^{\prime}}{\hbar^{2} / 2 m_{e}}\right)\left(1-\frac{m^{*}}{m_{e}}\right) \times \\
& \times \frac{1}{(1+\lambda)\left(1+\frac{2}{3} \lambda\right)}\left(\frac{m^{*}}{m_{e}}\right)\left(\frac{\varepsilon}{E_{g}}\right)^{3},
\end{aligned}
$$

where $\lambda=\Delta / E_{g}, \Delta$ is the spin-orbit splitting, $E_{g}$ is the energy gap and the parameter $A^{\prime}$ is defined as follows

$$
A^{\prime}=2 \frac{\hbar^{2}}{m_{e}^{2}} \sum_{u} \frac{\left\langle s\left|P_{x}\right| u\right\rangle\left\langle u\left|P_{y}\right| z\right\rangle}{E_{c}-E_{u}} .
$$

In the letter formula $s$ is a function transformed in accordance with $\Gamma_{1}$ irreducible representation of zincblende structure space group, $u$ is the periodic part of the Bloch function and $z$ is the function which transforms under the tetrahedral group transformations just like $P_{z}$ atomic functions .

According to G. Fishman and G. Lampel [12], $\Omega_{\mathbf{k}}^{2}(\varepsilon)$ for GaAs is approximately equal to $10^{18} \varepsilon^{3}$, if $\Omega$ is in $\operatorname{rad}$ per second and $\varepsilon$ is in $\mathrm{eV}$.
Since we deal with mesoscopic loop structure and 2DEG, it is also important to take into account another very similar mechanism of spin-splitting occurring in 2D electron gas and which was pointed out by $\mathrm{Yu}$. Bychkov and E. Rashba [10]. They noticed that in heterostructures and surface layers there is lack of inversion asymmetry due to the existence of interfaces. This type of asymmetry could be called SIA. The corresponding spinorbit Hamiltonian, according to [10] is of the form:

$H_{S O}=\xi[\sigma \times \mathbf{k}] \cdot v$,

where $\sigma$ are the Pauli matrices, $v$ is a unit vector perpendicular to the surface and $\xi$ is some constant whose numerical value can be established by the cyclotron resonance data.

As in previous case, the operator $H_{S O}$ lifts the twofold spin degeneracy at $\mathbf{k} \neq 0$ and determines the spin-orbit band splitting near $\mathbf{k}=0$.

Just in the same way as previously, this mechanism leads to the precession of spin axes and because of k-dependence and electron scattering, to the diffusion of the initial "spot" corresponding to the initial state of spin precession axis over entire $S^{2}$-surface. The frequency of the precession is equal to $\Omega_{\mathbf{k} v}=\Delta \varepsilon_{\mathbf{k} v} / \hbar$, where $\Delta \varepsilon_{\mathbf{k} v}$ corresponds to the spin splitting due to $H_{S O}$ (we add here subscript $v$ in order to distinguish this mechanism of spin precession from the first one).

It is clear, that if the semiconductor, of which the structure in question is made, is of zinc-blende type, we could expect these two mechanisms doing simultaneously together.

What is less obvious and which was first pointed out by P. Pfeffer and W. Zawadzki [13], that there is no simple additivity of these two mechanisms: $\Delta \varepsilon_{\text {tot }} \neq \Delta \varepsilon_{\mathbf{k}}+\Delta \varepsilon_{\mathbf{k} v}$ (subscript "tot" stands for "total"). According to [13], the $\Delta \varepsilon_{\text {tot }}$ depends on the subtleties of semiconductor band structure and electron density in 2DEG.

Now proceed to estimates of the spin relaxation time for the materials of zinc-blende type, such as GaAs, InSb, and InAs. To the authors knowledge, the most reliable data are known for the first of these three materials, so let us start from the estimates for GaAs. If one starts with the DP mechanism corresponding to BIA, then one can easily estimate $\Omega_{\mathbf{k}}(\mathrm{GaAs})$ as to be equal $\sim 3.088 \cdot 10^{11} \mathrm{~Hz}$. Taking into account SIA and its generalization proposed by P. Pfeffer and W. Zawadzki supposing the electron density in $2 \mathrm{DEG}$ to be equal $N_{s}=10^{12} \mathrm{~cm}^{-2}$, one get from the data of [13]. that $\Delta \varepsilon_{\text {tot }}(\mathrm{GaAs})$ is about $0.46 \mathrm{meV}$. Introducing $\Omega(\mathbf{k}, v)=\Delta \varepsilon_{\text {tot }} / \hbar$ and using the approach discussed above (see Eq. (4)) we can evaluate the spin relaxation time in the framework of the generalized model which includes both mechanisms. The time, according to our estimations, is approximately equal $T \approx 3.2 \cdot 10^{-8}$.

Using the data of [12], one can get for the ratios $\Omega_{\mathbf{k}}(\mathrm{GaAs}) / \Omega_{\mathbf{k}}(\mathrm{InSb})$ and $\Omega_{\mathbf{k}}(\mathrm{GaAs}) / \Omega_{\mathbf{k}}(\mathrm{InAs})$ the 
values: 0.378 and 1.58, respectively. As a consequence, $\Omega_{\mathbf{k}}(\mathrm{InSb}) \sim 8.037 \cdot 10^{11} \mathrm{~Hz}$ and $\Omega_{\mathbf{k}}(\mathrm{InAs}) \sim 1.216 \cdot 10^{11} \mathrm{~Hz}$ . Unfortunately, nothing is known for certain about SIA for these materials, as well as for its Pfeffer - Zawadzki generalization. Let us suppose, however, that the latter one leads to the same consequences for these two materials as in the previous case of gallium arsenide. Then, at $N=10^{12} \mathrm{~cm}^{-2}, \Delta \varepsilon_{\text {tot }}$ should be about twice as great as $\Delta \varepsilon_{\mathbf{k}}$, and the same is valid for $\Omega(\mathbf{k}, v)$. As a result, we have for $T(\mathrm{InSb}) \sim 2.4 \cdot 10^{-8} \mathrm{~s}$ and for $T($ InAs $) \sim 1.0 \cdot 10^{-6} \mathrm{~s}$. Of course, the latter results are only very rough estimates.

The natural question that is to be answered now is as follows: how does this spin relaxation time relate to the spin phase relaxation time $\tau_{\varphi}^{(s)}$ introduced above? One can relate the spin relaxation time $T$ to the phase relaxation time simply in the following way. Since our structure is in external magnetic field, the frequency of spin precession is the sum of $\Omega(\mathbf{k}, v)$ and the Zeeman frequency $\Omega_{z}=g \mu_{\mathrm{B}} B / \hbar: \Omega=\Omega(\mathbf{k}, v)+\Omega_{Z}$, where $g$ stands for the Lande factor. This frequency is a bit different for different electrons, since $\Omega(\mathbf{k}, v)$ depends on the electron quasi-momentum $\mathbf{k}$. Thus, the electron scattering means that the "oscillator" characterized by $\Omega$ is "triggering" all the time from the frequency $\Omega^{\prime}\left(\mathbf{k}^{\prime} v\right)+\Omega_{Z}$ to another one, $\Omega^{\prime \prime}\left(\mathbf{k}^{\prime \prime} v\right)+\Omega_{Z}$, than to $\Omega^{\prime \prime \prime}\left(\mathbf{k}^{\prime \prime \prime} v\right)+\Omega_{Z}$ and so on. Since each of these frequencies differ from one another only by some small value, each single scattering event leads only to small "phase aberration". Thus, the single "triggering" does not yet introduce the irreversibility and phase destruction. However, after many scattering events not only the direction of initial quasi-momentum changes, but its absolute value changes, too. The latter one introduces the necessary element of irreversibility and means the destruction of the "phase memory" in the spin part of electron wavefunction. The complete phase destruction occurs after the elapse of time $\tau_{\varphi, \mathrm{PZ}}^{(s)} \sim T \sim\left(\Omega_{\mathrm{tot}}^{2} \tau_{\mathbf{p}}\right)^{-1}$ (we add here the subscript "PZ", to emphasize that we used the generalized Pfeffer Zawadzki model).

Now compare the EY and precession mechanisms and estimate their combined influence on the spin phase relaxation.

To this end, let us make at first some additional comments concerning calculations of the spin phase relaxation time which is due to EY mechanism presented in Sec. 2. As we already mentioned, in Ref. [6] only the lower boundary for $\tau_{\varphi, \mathrm{ph}}^{(s)}$ was established, which nevertheless turned out to be much greater than $\tau_{\varphi}^{(e)}$. It

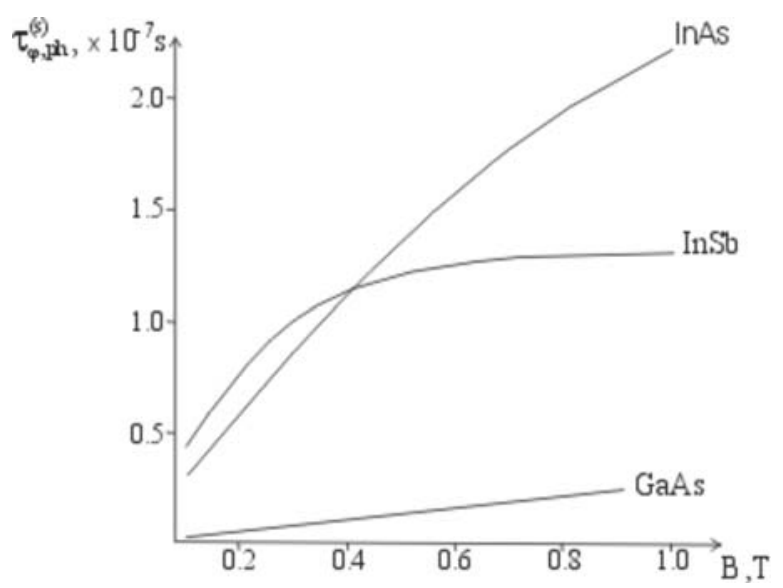

Fig. 3. Spin phase relaxation time due to Elliot-Yafet mechanism versus external magnetic field for three zinc-blende type semiconductors.

is possible to get more rigorous theory which could make more precise calculations possible. The theory could, for instance, utilize the spin-orbit operator of the form similar to that one used in [8], in which however, $U$-term should be substituted by $V_{q}(\mathbf{r})$, where $V_{q}(\mathbf{r})$ stands for the perturbation caused by a single phonon. Such theory is however beyond the scope of the paper, the more so that in this eventual theory there would be some other parameters that in their turn are not absolutely precise. Since our aim is only to show that $\tau_{\varphi, \text { ph }}^{(s)} \gg>\tau_{\varphi}^{(e)}$, we restrict our treatment rather to estimates. But in order to take into account the difference between GaAs, InSb and InAs, we include into $\varepsilon_{\text {int }}$ the factor $(\bar{Z} \alpha)$, where $\bar{Z}$ is the mean atomic number of the corresponding compound and $\alpha=e^{2} / \hbar c$. This factor is important, because the role of spin-orbit interaction increases as the atomic number $Z$ increases [11].

The results of our calculations are summed up in Fig. 3 and Table 1. In Fig. 3, the spin phase relaxation times which are due to EY mechanism are shown for $\mathrm{GaAs}$, InSb, and InAs as the function of external magnetic field. In Table 1, the spin phase relaxation times, due to EY mechanism and for two chosen values of magnetic field are compared to the relaxation times due to precession mechanism. Obviously, one can treat these mechanisms as independent and hence,

$\frac{1}{\tau_{\varphi}^{(s)}}=\frac{1}{\tau_{\varphi, \mathrm{ph}}^{(s)}}+\frac{1}{\tau_{\varphi, \mathrm{PZ}}^{(s)}}$.

Now it is clear that at least in accordance with our calculations, for GaAs the EY mechanism dominates for magnetic fields $B \leq 1 \mathrm{~T}$, because $\tau_{\varphi, p h}^{(s)}$ is the shortest time. For InSb one can conlude from the data of Table 1, that for small magnetic field precession mechanism 
dominates, because $\tau_{\varphi, \mathrm{PZ}}^{(s)}$ is the shortest time, while for $B \sim 1 \mathrm{~T}$ EY mechanism becomes dominating.

Table 1

\begin{tabular}{|c|c|c|c|}
\hline \multirow{2}{*}{ Semiconductor } & \multicolumn{2}{|c|}{$\tau_{\varphi, \mathrm{ph}}^{(s)}, \mathrm{s}$} & \multirow{2}{*}{$\tau_{\varphi, \mathrm{PZ}}^{(s), \mathrm{S}}$} \\
\cline { 2 - 3 } & $B=0.1 \mathrm{~T}$ & $B=1 \mathrm{~T}$ & \\
\hline $\mathrm{GaAs}$ & $2.6 \cdot 10^{-9}$ & $2.3 \cdot 10^{-8}$ & $3.2 \cdot 10^{-8}$ \\
\hline $\mathrm{InSb}$ & $4.27 \cdot 10^{-8}$ & $1.3 \cdot 10^{-7}$ & $2.4 \cdot 10^{-8}$ \\
\hline $\mathrm{InAs}$ & $2.9 \cdot 10^{-9}$ & $2.21 \cdot 10^{-7}$ & $1.0 \cdot 10^{-6}$ \\
\hline
\end{tabular}

It is interesting to compare our results with the experimental ones. According to the recent data obtained by D. Awschalom, J. Kikkawa and others [1, 4], the spin decay due to environmental decoherence can exceed even $100 \mathrm{~ns}$, and it is in excellent agreement with our calculations. So, again we can conclude that the condition $L_{\varphi}^{(s)}>L>L_{\varphi}^{(e)}$ can be satisfied.

\section{Transmission coefficient of the loop structure and the current}

The current $I$ through the structure considered in Sec. 2, for the small applied potential $V$, can be written as $[6,8]$ :

$$
\begin{aligned}
& I=\frac{2 e}{h} \int d E \int\left(w_{z} d k_{z} / 2 \pi\right) \times \\
& \times\left[f(E)-f(E+e V) \sum_{n^{\prime}, n^{\prime \prime}}\left|T_{n^{\prime}, n^{\prime \prime}}\right|^{2}\right] .
\end{aligned}
$$

Here $w_{z}$ is the width of the structure in the $z$-direction, $T_{n^{\prime}, n^{\prime \prime}}$ is the transmission coefficient from the state $n^{\prime}$ in the left-hand end to the state $n^{\prime \prime}$ in the right-hand end, $E$ and $k_{z}$ are the energy and the transverse wavevector of the electrons as they enter from the left-hand end.

The approach to calculation of the transmission coefficient $T_{n^{\prime}, n^{\prime \prime}}$ was outlined in [6]; here for reader's convenience, we add only few comments.

Since the length $L$ of the structure is supposed to be greater than $L_{\varphi}^{(e)}$, the key idea is to devide the structure into sections of the length smaller than $L_{\varphi}^{(e)}$. Then one can combine these successive scatters, considering the transport through the states $k^{\prime}, k^{\prime \prime}$ as incoherent, while the transport through the states $\sigma^{\prime}, \sigma^{\prime \prime}$ as coherent because of $L<L_{\varphi}^{(s)}$. As a result, the expression for the transmission coefficient is of the form (see [6]):

$$
T=t^{\prime}\left[I-P \cdot r \cdot P^{\prime} \cdot r^{\prime}\right]^{-1} P \cdot t,
$$

where the subscripts $\sigma^{\prime}, \sigma^{\prime \prime}$ are dropped out. Here $I$ is the unit matrix, $t$ is $4 \times 1$ matrix describing the transmission from the left-hand end into the two channels, while $t^{\prime}$ is $1 \times 4$ matrix describing the transmission from the channels into the right-hand end. Similarly, $r$ and $r^{\prime}$ are $4 \times 4$ matrices describing the reflections at the two junctions of the channels back into the channels. Matrices $P$ and $P^{\prime}$ describe forward and reverse propagation of the electron wave through the channels 1 and 2, respectively.

The general remark that should be made concerns the validity of the Landauer - Büttiker formalism in this particular case. It is known [14] that this formalism provides a rigorous framework for the description of mesoscopic transport as long as transport across the structure is coherent. For noncoherent transport, however, the situation is more complicated, if there is a "vertical flow" of electrons, that is the electron transitions from one energy to another. In that sence, our case is rather intermediate one: the transport through the states $\sigma^{\prime}, \sigma^{\prime \prime}$ is coherent, while through the states $k^{\prime}$, $k^{\prime \prime}$ is incoherent. Luckily, sometimes even if "vertical flow" is pressent, it can be neglected (see [14], p. 111) provided transmission functions are approximately constant over the energy range where transport occurs:

$\left(\mu_{1}+\mu_{2}\right)+\left(n / k_{\mathrm{B}} T\right)<<\varepsilon_{c}$,

where $1 \leq n \leq 5, \varepsilon_{\tau}$ is the correlation energy.

To estimate correlation energy, one can use simple relation: $\varepsilon_{c} \sim \hbar / \tau_{\varphi}^{(e)}$. Since $\tau_{\varphi}^{(s)} \gg>\tau_{\varphi}^{(e)} \sim 10^{-12}-10^{-13}$, correlation energy is about $0.6-6 \mathrm{meV}$. So, we assume the voltage $V$ applied to the structure to be sufficiently small, in order to satisfy the condition above.

Since we assume the length of the structure $L>L_{\varphi}^{(s)}$, there are no spin flips in two channels considered and hence, among the 16 matrix elements of $r$-matrix (as well as $\left.r^{\prime}\right) 8$ entries are equal to zero.

In order to construct $P$ and $P^{\prime}$, it is necessary to note that the spin parts of the wavefunctions acquire the phase factors due to Larmor spin precession around B-axis. Since magnetic fields in the channels are different, these phase factors are also different.

One can treat the states "spin up" and "spin down" as the two opposite points on a unit sphere $S^{2}$ which can be transformed one into another under rotation by an angle $\varphi= \pm \pi$ about some axis a. Introduce also formally b-axis which is a unit vector of the precession axis: $+\mathbf{b}$ corresponds to the electron propagation from $x=0$ to $x=L$ while $-\mathbf{b}$ corresponds to reverse propagation, as well as $\theta_{1}$ and $\theta_{2}$ are the phase acquired by spin part of the wavefunctions in the channels 1 and 2, respectively. Then the matrix elements describing the phase shifts in these two channels can be written as:

$$
\begin{aligned}
& P_{ \pm 1}=\exp \left( \pm i \varphi_{a}\right) \exp \left(i \theta_{1, b}\right), \\
& P_{ \pm 1}^{\prime}=\exp \left( \pm i \varphi_{a}\right) \exp \left(-i \theta_{1, b}\right), \\
& P_{ \pm 2}=\exp \left( \pm i \varphi_{a}\right) \exp \left(i \theta_{2, b}\right), \\
& P_{ \pm 2}^{\prime}=\exp \left( \pm i \varphi_{a}\right) \exp \left(-i \theta_{2, b}\right),
\end{aligned}
$$


The idea of Eqs (8), (9) is to express the elements of the matrices $P, P^{\prime}$ as two rotations about two independent axes. Then, these objects are nothing else but the unitary quaternions [15]. As is known [15], any quaternion can be written in the form

$q=c_{0}+i_{1} c_{1}+i_{2} c_{2}+i_{3} c_{3}=\Sigma_{\alpha=0}^{3} i_{\alpha} c_{\alpha}$,

where $i_{0}=1$ and $i_{1}^{2}=i_{2}^{2}=i_{3}^{2}=i_{1} i_{2} i_{3}=-1$. However, it is possible also to define, for instance, $i_{1}, i_{2}$ as

$i_{1}=\left(\begin{array}{cc}0 & 1 \\ -1 & 0\end{array}\right), i_{2}=\left(\begin{array}{cc}0 & i \\ i & 0\end{array}\right)$,

where $i$ is the ordinary complex square root of -1 , thus forcing

$i_{3}=i_{1} i_{2}=\left(\begin{array}{cc}i & 0 \\ 0 & -i\end{array}\right)$.

If these three matrices are multiplied by $-i$, one obtains the Pauli spin matrices. Thus, the quaternion $q$ could have been identified with the complex 2-by-2 matrix

$\left(\begin{array}{cc}c_{0}+i c_{3} & c_{1}+i c_{2} \\ -c_{1}+i c_{2} & c_{0}-i c_{3}\end{array}\right)=\left(\begin{array}{cc}u & v \\ -v^{*} & u^{*}\end{array}\right)$,

where $u$ and $v$ are complex numbers with complex conjugates $u^{*}$ and $v^{*}$. Replacing 0,1 , and $i$ in these complex matrices by

$\left(\begin{array}{ll}0 & 0 \\ 0 & 0\end{array}\right),\left(\begin{array}{ll}1 & 0 \\ 0 & 1\end{array}\right),\left(\begin{array}{cc}0 & 1 \\ -1 & 0\end{array}\right)$,

respectively, one can obtain a representation of quaternions as 4-by-4 matrices.

Since two channels 1 and 2 are supposed to be isolated, in this way the matrices $P$ and $P^{\prime}$ can be represented as the diagonal $4 \times 4$-matrices with the diagonal elements defined by Eqs (8), (9). After a great deal of algebra (see Ref.[6]), we have:

$$
\begin{aligned}
& |T|=\left|a_{1}\right|^{2}+\left|a_{2}\right|^{2}+\left|a_{3}\right|^{2}+\left|a_{4}\right|^{2}+ \\
& +\left(a_{1}^{*} a_{3}+a_{1} a_{3}^{*}+a_{2}^{*} a_{4}+a_{2} a_{4}^{*}\right)+ \\
& +\left(a_{1}^{*} a_{2}+a_{1} a_{2}^{*}+a_{2}^{*} a_{3}+a_{2} a_{3}^{*}+a_{3}^{*} a_{4}+a_{3} a_{4}^{*}\right) \times \\
& \times \cos \Delta \theta, \quad \Delta \theta=\theta_{1}-\theta_{2},
\end{aligned}
$$

where $a_{i}(i=1, \ldots, 4)$ do not depend on $\theta_{1}, \theta_{2}$ and are the complicated functions of $r_{i j}, r_{i j}^{\prime}, t_{i}, t_{i}^{\prime}$.

Note, that the cosine dependence of the transmission coefficient on phase difference in the latter expression is the direct consequence of the quaternion representation of the propagation matrices, $P$ and $P^{\prime}$.

It is interesting to note that the same cosine dependence on phase defference was obtained in the experiments with two interfering neutron beams [16], which are now considered as the direct verification of the $4 \pi$-symmetry of spinors.

\section{Calculation of the spin phase shift}

Consider the non-relativistic motion of the particle (electron) with the spin $|s|=1 / 2$ in a two-component magnetic field: $\mathbf{B}=\mathbf{B}_{0}+\mathbf{B}_{1}, \quad \mathbf{B}_{0}=\left(0, B_{0}, 0\right), \quad$ and $\mathbf{B}_{1}=\left(0,0, B_{1}\right)$, where $B_{1}$ is an additional uniform magnetic field in one of the channels of the structure. The spin part of electron wavefunctions can be considered as a two-component vector defined by the pair of functions $\chi(\mid \uparrow>)$ and $\chi(\mid \downarrow>)$ that stand for the probability amplitudes of the two possible orientations of spin. The spin operator $\hat{\sigma}\left(\sigma_{x}, \sigma_{y}, \sigma_{z}\right)$ is defined in terms of Pauli matrices:

$\sigma_{x}=\left(\begin{array}{ll}0 & 1 \\ 1 & 0\end{array}\right), \sigma_{y}=\left(\begin{array}{cc}0 & -i \\ i & 0\end{array}\right), \sigma_{x}=\left(\begin{array}{cc}1 & 0 \\ 0 & -1\end{array}\right)$

Thus, we can treat the mean value of the magnetic moment of the electron moving within the channels of mesoscopic structure as the classical quantity $\mathbf{P}=\langle\sigma\rangle$, its evolution under magnetic field being defined by the equation:

$\frac{d \mathbf{P}}{d t}=\gamma^{*}[\mathbf{P}, \mathbf{B}]$

where $\gamma^{*}=e / m c$ is the electron gyromagnetic constant.

In other words, the vector $\mathbf{P}$ can be treated as classical magnetic top and, if this classical top having the initial orientation $\mathbf{P}_{0}=\left(P_{x}^{0}, P_{y}^{0}, P_{z}^{0}\right)$ enters magnetic field $\mathbf{B}=\left(B_{x}, B_{y}, B_{z}\right)$, it begins to precess about magnetic field with the frequency $\Omega=\gamma^{*} B$, where $B=\sqrt{B_{x}^{2}+B_{y}^{2}+B_{z}^{2}}$.

It is interesting to note that despite its purely quantum character, the spin of the particle during its movement in external fields can be often treated classically. The accuracy of such treatment can be estimated by means of the Heisenberg uncertainty relation, since classical treatment is possible if one can neglect the commutator $[\mathbf{r}, \mathbf{p}]$, where $\mathbf{p}$ is the particle momentum operator. So, the measure of accuracy of the classical approximation is $|\Delta p| / p . \Delta p$ in our case can be estimated as $\sim m \Delta v=m\left(v^{2} / l_{\mathrm{B}}\right) \Delta t$, where $l_{\mathrm{B}}=\sqrt{\hbar c / e B}$ is the magnetic length and $\Delta t \sim 2 \pi / \omega_{c}$, $\omega_{c}=|e| B / m c$ is the cyclotron frequency, while $\Delta p / p \sim 2 \pi m v_{\mathrm{F}} \sqrt{m c} / \sqrt{\hbar e} B$.

Assuming $v_{\mathrm{F}} \sim 3 \cdot 10^{7} \mathrm{cms}^{-1}$ and $B \sim 0.1 \mathrm{~T}, \quad$ we have $|\Delta p| / p \approx 1.26 \cdot 10^{-10}$. Therefore, indeed to a good approximation, we can treat the evolution of vector $\mathbf{P}$ as the evolution of the classical magnetic top under external magnetic field.

Let us introduce now the phase of precessing spin by means of the formula 
$\Delta-\theta(v, x)=\mu_{B} g / \hbar \int_{0}^{x} B(v, x) d t=\gamma^{*} / v \int_{0}^{x} B d x^{\prime}$.

Since magnetic fields $\mathbf{B}_{0}$ and $\mathbf{B}_{1}$ are uniform, the calculation of the phase shift $\Delta \theta$ can easily be done.

Moreover, it is clear that under certain conditions including appropriate structure length $L$, electron velocity and the values of magnetic fields $B_{0}, B_{1}$, the phase shift $\Delta \theta=\theta_{2}-\theta_{1}$ can be multiple of $\pi$. Indeed,

$\Delta \theta=\theta_{2}-\theta_{1}=n \pi=(\gamma L / \nu)\left(\sqrt{B_{0}^{2}+B_{1}^{2}}-B_{0}\right), n=1,2 \ldots$

If the values of $B_{1}, L, v, n$ are given, the value of $B_{0}$ which is needed for the $\Delta \theta$ to be equal of multiple of $\pi$ can be easily calculated:

$B_{0}=\left|\frac{\gamma^{*} L}{2 n \pi \nu} B_{1}^{2}-\frac{n \pi \nu}{2 \gamma^{*} L}\right|$

Hence, changing the external magnetic field $B_{0}$, one can change the phase shift and the quantum interference from constructive to destructive one and back. Also it is seen that $\Delta \theta=\theta_{2}-\theta_{1}=f\left(B_{0}, B_{1}, v\right)$. That is, the phase shift generally speaking is different for the electrons with different velocities. At first sight, this makes matters worse, because it means that the "interference pattern" should be blurred. One should remember, however, that the temperature is considered to be sufficiently low. It means that most of the electrons carrying current are on the Fermi surface, that is the electron distribution function $f(\varepsilon)=\chi\left(\varepsilon_{\mathrm{F}}-\varepsilon\right)$ and $v=v_{\mathrm{F}}$, where $\chi(\ldots)$ is the Heaviside step-like function, $\varepsilon_{\mathrm{F}}, v_{\mathrm{F}}$ are the Fermi energy and Fermi velocity, respectively. Someone can be temptated to substitute $v$ in the latter formula by the drift velocity. But the condition $L_{\varphi}^{(s)}>L>L_{\varphi}^{(e)}$ does not imply that electrons undergo so many collisions that the drift velocity arises. Remember that the concept of drift velocity is relevant to macroscopic samples, where electrons undergo a great many collisions under which (and an external electric field) the drift velocity can only be formed. Here instead, we have mesoscopic structure where electrons suffer only a few collisions after which the phase coherence of the orbital part of the electron wavefunctions in the two arms of the structure is destroyed.

As a result, we can substitute in (10) $v$ by $v_{F}$ assuming that most of the electrons carrying current are on the Fermi surface and a few collisions which they undergo during their movement withing the channels of the structure do not change essentially their flight time which is still approximately equal to $\sim L / v_{\mathrm{F}}$.

So, the calculation by means of (10) taking into account the expression for $|T|^{2}$, now can easily be done and we have:

$I=(2 e / h) K\left(A+D \cos \Delta \theta\left(v_{\mathrm{F}}\right)\right)$, where $K, A, D$ are the coefficients dependent on the peculiarities of the structure. Now it is clear that changing $B_{0}$ one can approach very deep modulation of the conductance and since $A \sim D$, the "contrast" of the "interference pattern" is defined only by the ratio

$$
\sqrt{\frac{\varepsilon_{\mathrm{F}}-k_{\mathrm{B}} T}{\varepsilon_{\mathrm{F}}}} \text {. }
$$

So, we conclude that if the structure length $L$ is chosen to be $L_{\varphi}^{(s)}>L>L_{\varphi}^{(e)}$, it is indeed possible to "wash out" the quantum interference related to phase coherence of the „orbital part" of electron wavefunction, retaining at the same time that one related to the phase coherence of the spin part. Moreover, we can expect this "interference pattern" and corresponding current (or conductance) modulation to be strong enough in order to be observed.

It is also interesting to note that current (and conductance) oscillations generally speaking, are not periodic with respect to $B_{0}$, the magnetic field by means of which these oscillations are controlled. This is due to the fact, that while the second term in (10) is linearly proportional to $n$, the first one is inversely proportional to it. Another interesting feature of (10) is the quadratic dependence of $B_{0}$ on the static field $B_{1}$. By means of this formula one can easily calculate $\Delta B_{0}$, the changing in magnetic field $B_{0}$ which is needed to change $\Delta \theta$, for example, from $\pi$ to $2 \pi$. The corresponding data for three zinc-blende type semiconductors, two chosen values of $B_{1}$ and $L=1.5 \cdot 10^{-2} \mathrm{~cm}$ are presented in Table 2 .

\section{Table 2}

\begin{tabular}{|c|c|c|}
\hline \multirow{2}{*}{ Semiconductor } & \multicolumn{2}{|c|}{$\Delta B_{0}, \mathrm{~T}$} \\
\cline { 2 - 3 } & $B_{1}=0.1 \mathrm{~T}$ & $B_{1}=0.5 \mathrm{~T}$ \\
\hline $\mathrm{GaAs}$ & 0.002 & 0.91 \\
\hline $\mathrm{InSb}$ & 3.54 & 88.68 \\
\hline $\mathrm{InAs}$ & 1.07 & 26.75 \\
\hline
\end{tabular}

Keeping in mind the possibility of experimental verification of the theory presented in the paper, one can conclude from Table 2 that some materials and some values of magnetic field $B_{1}$ are more sutable than the others. Perhaps, GaAs is the best material for that purpose, while if $B_{1}>0.2 T$ such experiment for InSb and InAs becomes rather impossible.

\section{Quantum interference in time-dependent magnetic field (quantum beats)}

Whenever one deals with a physical phenomenon in which the motion of some object can be represented as the superposition of two harmonic oscillations with two angular frequencies $\omega_{1}, \omega_{2}$ which are very close to each other $\left(\omega_{1} \approx \omega_{2}\right)$, one can expect to observe the beats. That is, the resulting almost harmonic oscillation occurs 


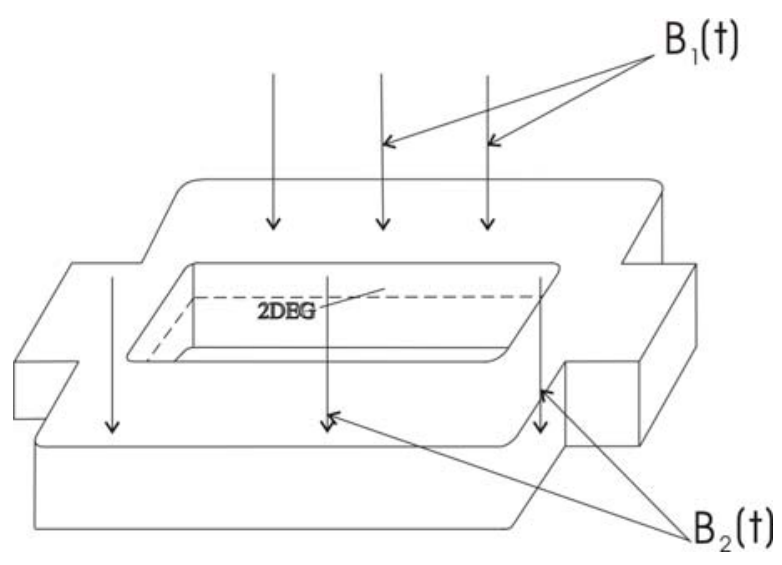

Fig. 4. Sketch of a two-channel semiconductor mesoscopic structure in external time-dependent magnetic fields of different amplitudes across the channels.

at the frequency $\varpi=1 / 2\left(\omega_{1}+\omega_{2}\right)$ and with the slowly varying amplitude, $A(t) \sim \cos \left(\omega_{\bmod } t\right)$, where $\omega_{\text {mod }}=1 / 2\left|\omega_{1}-\omega_{2}\right|$.

Such beats can be easily observed, for instance, in acoustics when the beats are induced by two vibrating tuning forks. If the frequencies of the forks vibrations, $\omega_{1}$ and $\omega_{2}$, differ by less than $10 \mathrm{~Hz}$, neither human ear nor brain can distinguish them and the human being perceives them as a single tone but with varying amplitude [17].

Beats in optics, as the beats between incoherent light sources, have been observed by A. Forrester, R. Gudmundsen and P. Johnson [18]. In their very nice experiments, they mixed up two Zeeman components of a visible spectral line of $\mathrm{Hg}$ atom. The mean frequency of corresponding lines was about $5.49 \cdot 10^{14} \mathrm{~Hz}$, while the frequency difference in the experiments of [18] was about $10^{10} \mathrm{~Hz}$, which corresponds to the microwaves.

Consider again the loop srtucture similar to that one of Sec. 2. The main difference however now is that an external magnetic field is time-dependent and its amplitude is supposed to be somewhat different in the channels 1 and 2 (Fig. 4).

Suppose the Hamiltonian of an electron is (see also [19]): $H(t)=H_{0}(t) \otimes I_{0}+H_{1}(t) \otimes I_{1}$, where

$$
\begin{aligned}
& H_{0}=1 / 2 m^{*}(\mathbf{p}-(e / c) \mathbf{A}(t))^{2}+\mathbf{U}(\mathbf{r}), \\
& H_{1}=-\mu_{\mathrm{B}} \hat{\sigma} \cdot \mathbf{B}(t) .
\end{aligned}
$$

Here all the denotions are as in Sec. 2. Assume the characteristic time scale of magnetic field changing is much longer than all characteristic electron scattering times, spin relaxation time including. Then the main results of Refs $[6,8]$ are applicable to this particular case of time-dependent magnetic field. Consider now the non-relativistic motion of an electron with the spin $|s|=1 / 2$ in a loop structure of Fig. 4, where the magnetic fields in the two arms of the structure are equal: $B_{1}=B_{01} \cos \omega \mathrm{t}$ and $B_{2}=B_{02} \cos \omega \mathrm{t}$. In accordance
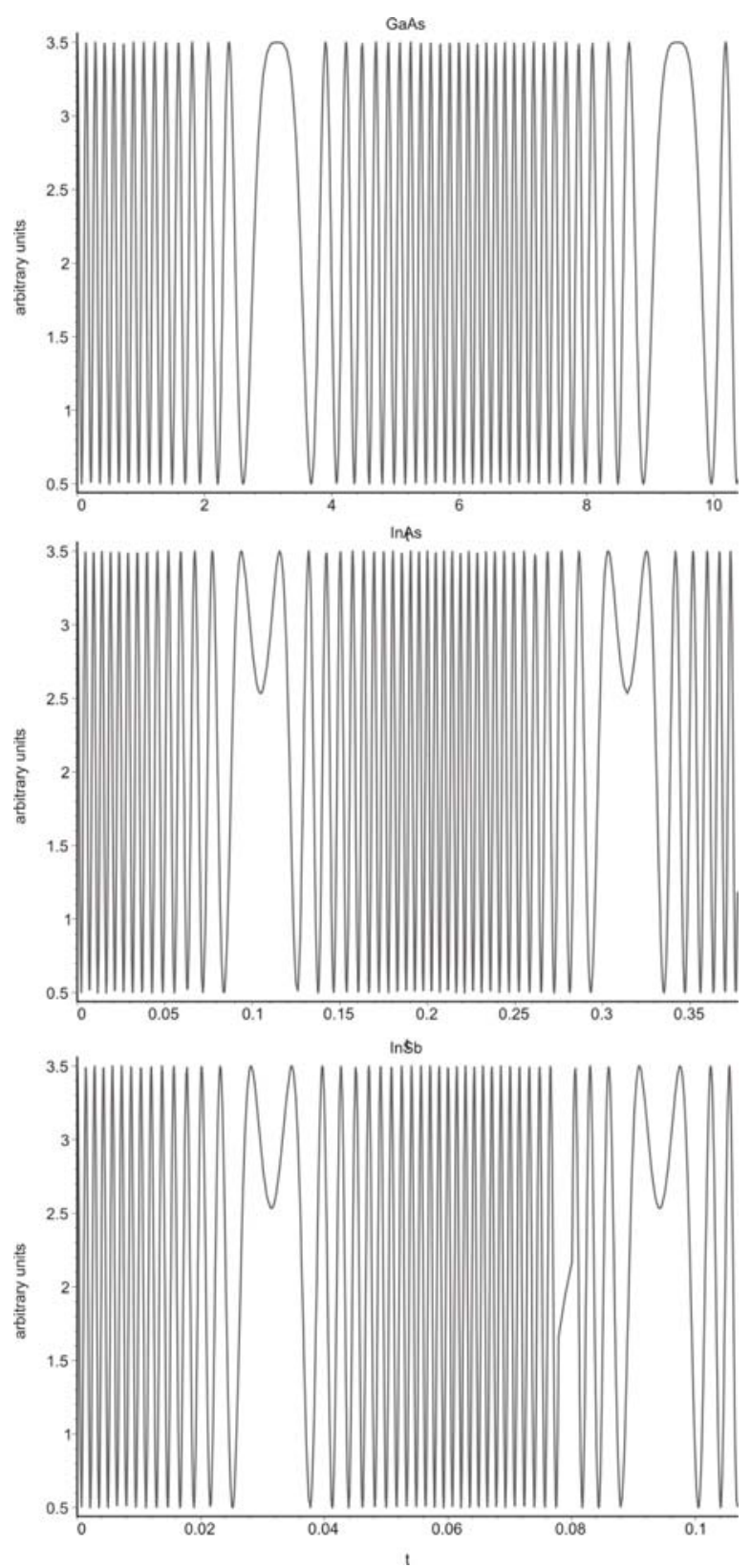

Fig. 5. Quantum beats in mesoscopic loop structure due to spin coherent transport and Larmor precession. Three pictures represent current through the structure versus time for three different semiconductors. The parameters chosen for the calculations are: InSb $-|g|=50.7, \omega=50 \mathrm{~Hz}$; InAs $|g|=15.3, \omega=15 \mathrm{~Hz} ; \mathrm{GaAs}-|g|=0.50, \omega=0.5 \mathrm{~Hz}$; $\Delta B=10^{-5}$ Gs throughout.

with general quantum mechanical approach (see, for instance, $\mathrm{AB}$ phase description given by R. Feynman [20]), time-dependent phase of precessing spin (actually, the Larmor rotating angle around the field B) can be introduced as follows: 
$\theta(t)=\left(\mu_{\mathrm{B}} g / \hbar\right) \int_{0}^{t} B\left(t^{\prime}\right) d t^{\prime}$

Here we again suppose the majority of electrons to be at the Fermi surface and to have the velocity equal to the Fermi velocity $v_{\mathrm{F}}$.

Then using technique described in previous sections, one can calculate the current through the structure, which is equal to:

$$
I=(2 e / h) K(A+D \cos (\Delta \theta(t))) \text {, }
$$

where $K, A, D$ are the coefficients dependent on the peculiarities of the structure and related to $a_{i}(i=1, \ldots, 4)$, mentioned in Sec. 4 . The phase shift $\Delta \theta(t)$ acquired by the spin wavefunction of an electron moving through the structure, is:

$\Delta \theta(t)=\left(\mu_{\mathrm{B}} g \Delta B / \hbar \omega\right) \sin \omega t$,

where $\Delta B=B_{01}-B_{02}$.

Fig. 5 represents the current through the loop structure plotted according to the formulae (13), (14) for different semiconductors and some chosen values of $\Delta B$ and $\omega$; we also assumed $A \sim D$. It is clearly seen that in all cases the curves representing current versus time consist of a spire-like pieces separated by rather more slow undulations. Obviously, spire-like pieces correspond to relatively great values of the prefactor $\left(\mu_{\mathrm{B}} g \Delta B / \hbar \omega\right)$ in (14) and the values of sin $\omega t$ which are very close to unity, while relatively slow undulations correspond to those values of $\sin \omega t$ that are very nearly to zero. Therefore, if one would measure the current through the structure in question by means of the device with relatively rough time resolution, this fine spire-like structure would be smeared out and one could observe only that current undulates up and down from its average value almost periodically. From Fig. 5, one can easily estimate this periodicity: if one has the device which can probe the current with time resolution $0.01 \mathrm{~s}$, then the period of undulations for InSb would be about $0.06 \mathrm{~s}$, for InAs $-0.05 \mathrm{~s}$ and for GaAs - about $6 \mathrm{~s}$ (notice, that $\Delta B$ in all cases is supposed to be the same, while $\omega$ is chosen to be different in order to "compensate" the difference in Lande factor $g$ for these three materials).

It is especially easy to grasp what is going on, if one suppose that the frequency of magnetic field oscillations $\omega$ is so small that the condition $\omega t<<1$ is fulfilled. Then, from Eqs (13), (14) we have

$$
I \propto\left(A+D \cos \left(\left(\mu_{\mathrm{B}} g \Delta B / \hbar\right) t\right)\right) .
$$

It is clear, that since the factor $\omega_{0}=\mu_{\mathrm{B}} g \Delta B / \hbar$ in the latter expression, generally speaking, is large enough (for example, if $\Delta B \sim 1 \mathrm{~T}, \quad \omega_{0} \sim 4.4 \cdot 10^{10} \mathrm{~Hz}$ ), on average the current through the structure is very nearly to some constant. However, if $\Delta B$ becomes very small, say, $10^{-5}-10^{-6} \mathrm{Gs}$, the period $T=2 \pi / \omega_{0}$ of oscillations in (13) becomes equal to $0.14-1.4 \mathrm{~s}$ and this yields the possibility to observe current modulation.
Thus, the current through the structure should oscillate without any apparent change in the structure and this kind of oscillations can also be called quantum beats. These beats are very similar to the Josephson effect. Indeed, in this case the phase difference $\Delta \theta(t)$ is driven by the magnetic field (see Eqs (14), (15)) which is analogous to the Josephson effect in superconducting tunnel junctions, where the phase of Cooper pairs in two superconductors separated by the insutator film (socalled weak link) is related according to $\Delta \theta(t)=(2 \mathrm{eV} / \hbar) t$, where $V$ is the voltage applied to the junction and the superconducting Josephson current of

$$
I_{S}=I_{S \max } \sin \Delta \theta(t) \text {. }
$$

Let us analize an amazing similarity of these two effects more thoroughly. Indeed, while the phase difference of the Cooper pairs in the Josephson junction driven by an applied voltage is equal $\Delta \theta(t)=2 \mathrm{eVt} / \hbar$, the phase differens of the electrons spin wavefunction acquired during electron transport through the channels of the loop structure driven by magnetic field, is equal to $\Delta \theta(t)=\mu_{\mathrm{B}} g \Delta B t / \hbar$. The effect discussed here differs, however, from Josephson one in some important respect. In order to grasp it, let us look at the effect discussed in the paper, from another point of view. Namely, from the the view point of its possible applications to measuring the extremely small magnetic field deviations from the spatial uniformity.

It is well known that for many years superconducting quantum interference devices (SQUIDs) operating at $4 \mathrm{~K}$ have been unchallenged as ultrahigh-sensitivity magnetic field detectors [21]. They have enabled, for instance, biomagnetic imaging, such as mapping of the heart activity, mapping of the magnetic fields produced by the brain and so on. Since the current through the single SQUID (that is, two Josephson junctions in parallel, making a superconducting loop) is proportional to $\cos |2 e \Phi / \hbar|$, where $\Phi$ is the magnetic flux threading the superconducting loop, one has, in order to enhance sensitivity of the device, to increase the flux and hence, the area of the loop. To this end one has to put a set of 10,20 or even more Josephson junctions close together and equally spaced. Note that $2 e \Phi / \hbar$, is nothing else but the $\mathrm{AB}$ phase acquired by the Cooper pairs during their transport along the superconducting loop. Now it is clear in what respect the effect discussed in the paper differs from the $\mathrm{AB}$ one. The phase difference acquired by the electron spins does not depend on the flux threading the loop, but does depend on the magnetic field difference in the channels 1 and 2. It means that this effect perhaps could be used for constructing the devices complementary to SQUIDs in that respect that they could enable to measure the slightest magnetic field deviations from the spatial uniformity in extremely small scale. How small the measuring area could be, one can estimate in a following way. In order to satisfy the condition $L_{\varphi}^{(s)}>L>L_{\varphi}^{(e)}$, it is sufficient to get $L \sim 1.5 \cdot 10^{-2} \mathrm{~cm}$, while the distance between the 
channels 1 and 2 could be about $4.0 \cdot 10^{-6} \mathrm{~cm}$ with the width of the channel of about $1.0 \cdot 10^{-6} \mathrm{~cm}$ each. Then the measuring area can be about $9.0 \cdot 10^{-8} \mathrm{~cm}^{2}$; compare this value with the millimeter-scale spatial resolution of the SQUIDs or even with a somewhat smaller spatial resolution of a recently reported subfemtotesla atomic magnetometer [22]. The sensitivity of the device based on the effect discussed here, can be estimated as to be $10^{-11} \mathrm{THz}^{-1 / 2}$, while its operating temperature could be about $40 \mathrm{~K}$ or even more. Indeed, since in the formula (11) $A \sim D$, the "contrast" of the "interference pattern" is determined only by the ratio $\sqrt{\left(E_{\mathrm{F}}-k_{\mathrm{B}} T\right) / E_{\mathrm{F}}}$ which at a temperature of about $40 \mathrm{~K}$ is of the order of $90 \%$.

It is also instructive to compare the effect discussed in the paper, with other cyclic phenomena such as scalar Aharonov - Bohm (SAB) [23] and Aharonov - Casher (AC) [24] effects.

It is well known that in the $A B$ effect a charge moving around magnetic flux filament in a region with vanishing electromagnetic fields, accumulates the phase shift. This is due to gauge invariant coupling between the current and electromagnetic vector potentail and for that reason, the locally accumulated phase is not gauge invariant. Therefore, the $\mathrm{AB}$ effect is sometimes termed as being nonlocal. On the other hand, in the SAB effect, as it was argued by M. Peshkin [23] and in the AC effect, as it was shown in [25], the magnetic moment of a neutral particle couples directly to the field strenghts, either $\mathbf{B}$ (as in the case of SAB) or to $\mathbf{E}$ (as in the case of AS).

The effect discussed here is very similar to SAB and $\mathrm{AC}$, besause it is also brought about by an ordinary action of the Maxwell field and hence has the properties of all other local interactions. The AB effect is nonlocal in that the electron experiences no force and exchanges no momentum, energy or angular momentum with the electromagnetic field. In our case, just like in case of $\mathrm{SAB}$ and $\mathrm{AC}$, the Hamiltonian and the equation of motion involve contemporaneous Maxwell field in the domain of the electron position; thus, the effect is not entirely topological in its character. The main difference between this effect and $\mathrm{SAB}$ and $\mathrm{AC}$ is that the former deals with charged particles (electrons) in semiconductor and could be observable only under special conditions, when the "phase memory" related to the orbital part of the wavefunction is "washed out", while the phase coherence of the spin part of the wavefunction remains intact. Thus, this spin coherence can reveal itself in corresponding spin current oscillations.

\section{Conclusions}

A simple theory of the quantum interference due to Larmor precession of an electron spin in a loop structure is presented in this paper. We investigate different mechanisms of environmental decoherence, such as the edge scattering, the EY and precession mechanisms of spin relaxation, as well as their influence on the quantum spin interference in such structure. It turns out, that the time of spin phase relaxation due to edge scattering is very long and this mechanism can be neglected, while the other two are essential. The EY and precession mechanisms thus determine the spin phase relaxation. As it is shown, even if the EY and precession mechanisms do together, it is still possible nevertheless, to satisfy the condition $L_{\varphi}^{(s)}>L>L_{\varphi}^{(e)}$. The latter one determines the "spin ballistic" transport in the structure in question, that is, the phase relaxation length $L_{\varphi}^{(s)}$ of the spin part of the electron wavefunction is assumed to be greater than the microstructure length. If in one of the microstructure arms there is an additional magnetic field, the spin wavefunction acquires a phase shift due to additional spin precession around that field. Now, if we suppose the microstructure length is chosen to be greater than the $L_{\varphi}^{(e)}$, it is possible to "wash out" the quantum interference related to phase coherence of the "orbital" part of the wavefunction retaining at the same time that related to the phase coherence of the spin part and hence, reveal the corresponding conductance oscillations. Changing the external magnetic field, one can change the "interference pattern", that is, to control the conductance modulation. We have shown that the strong conductance modulation can be achieved in this way.

We also considered the spin interference in timedependent magnetic field and quantum beats in the loop structure that also occurr due to Larmor precession of an electron spin. We have shown that if the amplitudes of magnetic fields in the channels 1 and 2 of the loop structure are a little bit different, say $\Delta B \sim 10^{-5}-10^{-6} \mathrm{Gs}$, the quantum beats reveal themselves as the current modulation with a period depending on $\Delta B$ as well as on the material which the structure is made of. The last one could be used for developing a device complementary to SQUIDs, which could make possible the measuring of extremely small deviations of magnetic fields from their spatial uniformity. It could be used perhaps for biomagnetic imaging, such as heart and brain activity mapping and so on.

\section{References}

1. D.D. Awschalom, D. Loss, N. Samarth (Eds.) Semiconductor spintronics and quantum computation, Springer, Berlin, NY (2002).

2. Hong X. Tang, F.G. Monzon, Friso J. Jedema et al. // Ibid.

3. D. Hägle, M. Oestreich, W.W. Rüle et al. // Appl. Phys. Lett. 1580 (1998).

4. D.D. Awschalom, J.M. Kikkawa // Physics Today 33 (1999); J.M. Kikkawa, J.A. Gupta, I. Malajovich, D.D. Awschalom // Physica E 194 (2001).

5. S. Washburn, R.A. Webb // Adv. in Phys. 375 (1986).

6. I.Tralle // J. Phys.: Condens. Matter 8239 (1999). 
7. I. Tralle, W. Paśko // In: Symmetry and structural properties of condensed matter, Proc. 7th Intern. School on Theor. Phys., World Scientific, Singapore (2003).

8. I. Tralle, W. Paśko, Low-dimentional systems and nanostructures // Physica E 336 (2003).

9. M.I. D'yakonov and V.I. Perel'// Fiz. Tverd. Tela 3851 (1981)[Sov. Phys.-Solid State 3023 (1972)].

10. Yu.A. Bychkov, E.I. Rashba // J. Phys. C 6039 (1984).

11. V.F. Gantmacher, I.B. Levinson, Carrier scattering in metals and semicoductors. North-Holland, NYTokyo (1987).

12. G. Fishman, G. Lampel // Phys. Rev. B 820 (1977).

13. P. Pfeffer, W. Zawadzki // Ibid. R14 332 (1995).

14. S. Datta, Electronic transport in mesoscopic systems. Cambridge University Press, Cambridge (1995).

15. G. Casanova, L'algebre vectorielle. Presses Universitaires de France, Paris (1976).

16. H. Rauch // Europhysics News 10 (1997).

17. F.S. Crawford, Jr, Waves, Berkeley Physics Course. Vol. 3, McGraw-Hill (1965).
18. A.T. Forrester, R.A. Gudmundsen, P.O. Johnson // Phys. Rev. 1691 (1955).

19. I. Tralle, W. Paśko // Phys. Lett. A 463 (2003).

20. R. Feynman, R. Leighton, M. Sands, The Feynman Lectures on Physics. Vol. 2, Addison-Wesley, NY (1965).

21. H.Weinstok (ed.) SQUID Sensors:Fundamentals, Fabrication and Applications. Kluwer Acad., Dordrecht (1996).

22. I.K. Kominis, T.W. Kornack, J.C. Allred, M.V. Romalis // Nature 574 (10 April, 2003).

23. A.G.Klein, in: Fundamental Problems in Quantum Theory: a Conference Held in Honor of Professor John Wheeler (Annals of New York Acad. of Sci., vol. 755). Ed. by D.M. Greenberg and A. Zeilinger, New York Acad. of Sci.(1996); M. Peshkin, ibid.

24. Y. Aharonov and A. Casher // Phys. Rev. Lett. 319 (1984).

25. A.V. Balatsky, B.L. Altshuler // Ibid. 13441 (1993). 